\title{
Polycystic Ovary Syndrome, Subfertility and Vitamin D Deficiency
}

\author{
Arfa Azhar ${ }^{1}$, Fatima Abid ${ }^{2}$ and Rehana Rehman ${ }^{1}$ \\ ${ }^{1}$ Department of Biological and Biomedical Sciences, The Aga khan university, Karachi, Pakistan \\ ${ }^{2}$ Department of Physiology, Jinnah Sindh Medical Institute, Karachi, Pakistan
}

\begin{abstract}
Polycystic ovarian syndrome (PCOS) is the most common endocrine female disorder, affecting 4-18\% women of reproductive age. The prevalence of PCOS in South Asian women, especially in Pakistani women, is much higher (52\%) as compared to white population (20 - 25\% in UK). In Pakistan, vitamin D status displays 31.2\% vitamin D insufficiency, 53.5\% vitamin D deficiency, and $15.3 \%$ normal vitamin D values. Vitamin D deficiency, though very common in the general population, is even more prevalent in PCOS patients, seen in approximately $67-85 \%$, which further correlates to their related comorbidities.

A lot of researches have to be directed to examine the association among the PCOS and vitamin D, which may display monitoring role in several symptoms related to PCOS, such as ovulatory dysfunction, endocrine disruption, and insulin resistance. Vitamin D supplementation can, therefore, be employed to improve the metabolic and endocrine disorders; especially improving hormonal profile, oxidative stress, and ovulation outcome in PCOS patients.
\end{abstract}

Key Words: Vitamin D, Infertility, Ovulatory Dysfunction, Insulin Resistance.

How to cite this article: Azhar A, Abid F, Rehman R. Polycystic Ovary Syndrome, Subfertility and Vitamin D Deficiency. J Coll Physicians Surg Pak 2020; 30(05):545-546. DOI: https://doi.org/10.29271/jcpsp.2020.05.545.

The exact clinical features of polycystic ovary syndrome (PCOS) comprise clinical or biochemical hyperandrogenism, oligomenorrhea or amenorrhea, and polycystic ovarian morphology that could cause chronic anovulation and infertility. Presence of 2 out of 3 features is diagnostic as contemplated by European Society of Human Reproduction and Embryology (ESHRE) guidelines. ${ }^{1}$

Women with PCOS present a relatively high prevalence of vitamin D deficiency (VDD) incomparison with the general population, and this deficiency correlates with comorbidities associated with PCOS. Hormonal abnormalities triggered by steroidogenesis irregularities appear to be the key culprits of the clinical picture in PCOS. Some evidences have revealed that disturbances in steroidogenesis pathway, initiated by excessive androgen or insulin resistance (IR) in PCOS women, to be a prominent element of ovarian dysfunction and anomalous folliculogenesis. In vitro and in vivo studies demonstrated that the vitamin $D$ pathway might have a regulatory role in several PCOS associated symptoms, including ovulatory dysfunction, endocrine alterations, and IR. ${ }^{1}$ Vitamin $D$ encourages production of estradiol, estrone and progesterone, in human ovarian cells, both independently and, in the case of estradiol, along with insulin. ${ }^{2}$

Correspondence to: Dr. Rehana Rehman, Department of Biological and Biomedical Science, The Aga Khan University Hospital, Stadium Road, Karachi, Pakistan E-mail: rehana.rehman@aku.edu

Received: February 21, 2020; Revised: May 28, 2020;

Accepted: May 28, 2020

DOI: https://doi.org/10.29271/jcpsp.2020.05.545
PCOS patients have excessive luteinizing hormone (LH) and depleted follicle-stimulating hormone (FSH), which cause increased androgen synthesis that leads to development of IR, hyperinsulinemia and ovarian dysfunction. ${ }^{3}$

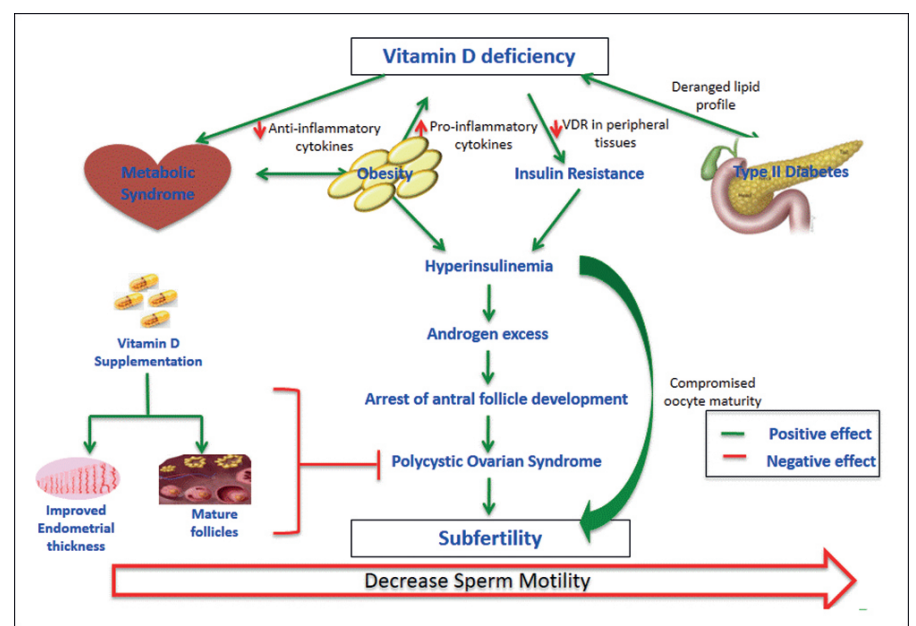

Figure 1: Impact of vitamin deficiency on fertility.

Vitamin D shows a foremost part in modulating multiplicative development of females by regulating steroidogenesis of sex hormones, as supported by the manifestation of vitamin D receptor (VDR) and 1-hydroxylase in ovaries, uterus, placenta, pituitary and hypothalamus. VDD has been shown to play a significant part in the progress of IR. It appears that VDD may lead to hyperandrogenism and insulin resistance in PCOS females by diminishing steroid hormone binding globulin (SHBG) intensities and insulin receptors, individually.

Additionally, it has been found out that females with PCOS have raised intra-follicular and circulating anti-Mullerian hormone $(\mathrm{AMH})$, usually formed by the granulosa cells of developing 
follicles, due to rise in the quantity of follicles, besides hyper secretion through granulosa cells themselves. ${ }^{4}$ Vitamin D normalises the secretion of $\mathrm{AMH}$ and also affects follicle selection by diminishing AMH mRNA expression. Furthermore, in vitro treatment of granulosa cells with 1, 25-dihydroxyvitamin D3 improves progesterone secretion from the precursor pregnenolone, ${ }^{3}$ as illustrated in Figure 1. Likewise, it has been proved by in vivo studies that vitamin D amplifies estrogen, progesterone, estrone, and insulin-like growth factor-binding protein 1 secretion in female ovarian cells. ${ }^{5}$

At the same time, 1, 25-dihydroxyvitamin D3 diminishes the appearance of AMH II and FSH receptor mRNA with raised secretion of progesterone in primary human granulosa cells (Figure 1). This indicates that Vitamin D changes granulosa cell diversity and results in luteinization accompanied by an associated rise in progesterone secretion that can lead to atresia and apoptosis of follicles in PCOS females. Therefore, this situation indicates that increased sensitivity to AMH levels in PCOS patients causes decreased progesterone secretion, which causes apoptosis of granulosa cells by preventing aromatase enzyme essential for estrogen production in the vesicles. ${ }^{6}$ In addition, VDD decreases the chances of acquiring endometrial thickness required for implantation of embryo.

New interventional trials have shown that adequate vitamin $D$ upswing in females with vitamin D insufficiency leads towards improvement in their deteriorated fertility and metabolic hormone profiles, reinforcing the importance of the vitamin D grading in diet, etiology and management of PCOS[RR1] . It has been demonstrated that it is necessary to screen for vitamin $D$ status in all patients with PCOS.

On account of association between VDD in PCOS and expected role in fertility, we suggest further research and trials to confirm the usefulness of vitamin D supplementation.

Vitamin D normalises hormone secretion and receptor appearance in granulosa and theca cells of growing follicles. It comprises of follicle deployment and maturation and fertilisation.
These are abbreviations (3 $\beta H S D$, 3- $\beta$-hydroxysteroid dehydrogenase; $\mathrm{AMH}$, anti-Mullerian hormone; $\mathrm{AMHR}$, anti-Mullerian hormone receptor; E2, estrogen; FSHR, follicle-stimulating hormone receptor; $\mathrm{LH}$, luteinizing hormone; P4, progesterone; (VDRE).

\section{CONFLICT OF INTEREST:}

Authors declared no conflict of interest.

\section{AUTHORS' CONTRIBUTION:}

$A A$ : Conceived the idea and did literature search.

FA: Helped in literature search and write-up.

RR: Reviewed the write-up.

\section{REFERENCES}

1. Qazi I, Qazi A, ljaz F, Jawed S, Aftab R, Qazi S. Relationship of obesity with insulin resistance in polycystic ovarian syndrome. Pak J Physiol 2018; 14:46-9.

2. Parikh G, Varadinova M, Suwandhi P, Araki T, Rosenwaks Z, Poretsky $L$, et al. Vitamin $D$ regulates steroidogenesis and insulin-like growth factor binding protein-1 (IGFBP-1) production in human ovarian cells. Horm Metab Res 2010; 42:754-7.

3. Merhi Z, Doswell A, Krebs K, Cipolla M. Vitamin D alters genes involved in follicular development and steroidogenesis in human cumulus granulosa cells. J Clin Endocrinol Metab 2014; 99:E1137-45.

4. 4.Pellatt L, Rice S, Dilaver N, Heshri A, Galea R, Brincat M, et al. Anti-müllerian hormone reduces follicle sensitivity to follicle-stimulating hormone in human granulosa cells. Fertil Steril 2011; 96:1246-51.

5. Parikh G, Varadinova M, Suwandhi P, Araki T, Rosenwaks Z, Poretsky L, Seto-Young D. Vitamin $D$ regulates steroidogenesis and insulin-like growth factor binding protein-1 (IGFBP-1) production in human ovarian cells. Horm Metab Res 2010; 42:754-7.

6. Muscogiuri G, Altieri B, de Angelis C, Palomba S, Pivonello R, Colao A, et al. Shedding new light on female fertility: The role of vitamin D. Rev Endocr Metab Disord 2017; 18:273-83. 